\title{
Optoelectronic characterization of carrier extraction in a hot carrier photovoltaic cell structure
}

\author{
James A. R. Dimmock ${ }^{1,2}$, Matthias Kauer ${ }^{1}$, Katherine Smith ${ }^{1}$, Huiyun Liu ${ }^{3}$, \\ Paul N. Stavrinou ${ }^{2}$, Nicholas J. Ekins-Daukes ${ }^{2}$ \\ ${ }^{1}$ Sharp Laboratories of Europe Ltd, Oxford, OX4 4GB, UK \\ ${ }^{2}$ Blackett Laboratory, Imperial College London, SW7 2AZ, UK \\ ${ }^{3}$ Department of Electronic and Electrical Engineering, University College London, WC1E 7JE, UK \\ E-mail: james.dimmock@sharp.co.uk
}

\begin{abstract}
A hot carrier photovoltaic cell requires extraction of electrons on a timescale faster than they can lose energy to the lattice. We optically and optoelectronically characterize two resonant tunneling structures, showing their compatability with hot carrier photovoltaic operation, demonstrating structural and carrier extraction properties necessary for such a device. In particular we use time resolved and temperature dependent photoluminescence to determine extraction timescales and energy levels in the structures and demonstrate fast carrier extraction by tunneling. We also show that such devices are capable of extracting photo-generated electrons at high carrier densities, with an open circuit voltage in excess of $1 \mathrm{~V}$.
\end{abstract}

Keywords: Hot Carrier Solar Cell, Resonant Tunneling Devices, Photoluminescence

\section{Introduction}

The hot carrier solar cell is a heat engine; supplementing or supplanting the photovoltaic action of a traditional solar cell with a thermally driven current, analogous to a thermoelectric device [1]. With this additional channel for energy extraction it is, in principle, possible for these cells to achieve efficiencies up to $85 \%$ [2], since the thermalisation loss of high energy carriers is mitigated through their contribution to the heat current.

The driving force for an operational hot carrier photovoltaic cell is, therefore, a difference in temperature between the region in which carriers are photo-generated and the region to which they are extracted. Maintaining these two regions (nominally the "absorber" and the "collector" from here on) at different temperatures is the primary goal of any device attempting to be a hot carrier photovoltaic cell. While it is not incompatible with theory that such a device could be realized based on differences in lattice temperature, the engineering challenge in maintaining two regions in electrical contact at an optimal temperature difference of thousands of Kelvin [3] is probably insurmountable. For this reason the hot carrier solar cell maintains this temperature differential in the electron population rather than in the phonon population. This necessitates that a good hot carrier solar cell must have an electrical transport path between the absorber and collector that allows transfer of electrons at a rate faster than the electrons can interact with the lattice. Furthermore, this electronic transport path must be energy selective in order to minimise transfer of heat to the collector and to the contacts [4].

In order to realize such a structure various groups have investigated methods to slow the rate of energy decay from the electron population, both through the use of quantum confined structures [5] and through materials choices that reduce phonon decay [6], with this latter method primarily aimed at identifying and developing materials that aim to frustrate the Klemens decay mechanism, though other decay paths are equally important [7].

In previous work [8], we have shown a hot carrier photovoltaic cell which approached this problem through fast extraction, rather than slowed carrier thermalisation. This work showed a hot carrier photo-current, which was dependent on the wavelength of light used to illuminate the cell, demonstrating the extraction of carriers prior to their thermalisation. The structure used was based on ultra-fast extraction of carriers from a narrow band gap semiconductor absorber to a wider band gap semiconductor collector through an energy selective tunneling contact. While this previous structure provided an important proof of principle, showing hot carrier electrical extraction, as a solar cell it was limited by a low open circuit voltage $\left(\mathrm{V}_{\mathrm{oc}}\right)$ and the carrier transport was not directly explored.

In the present work we explore this device concept further by investigating the structural and carrier transport properties of two related structures. The first structure investigated (structure $\mathrm{A}$ ) has an increased $\mathrm{V}_{\mathrm{oc}}$ by using a $\mathrm{p}-\mathrm{i}-\mathrm{n}$ structure rather than an $n-i-n$ structure as used in [8]. The second structure (structure B) is structurally similar to structure A but has a multiple quantum well (MQW) region embedded in the wider band gap collector to provide a signature region for photoluminescence (PL) characterization of the device.

In the following section we will outline the conceptual aim behind developing and examining these two samples, their sample design and fabrication, followed by low temperature current-voltage (IV) analysis of structure A (the p-i-n 
structure) in section III. We will then present a photoluminescence investigation of structure B (the MQW structure), with section IV exploring its temperature dependent PL (TDPL) in order to determine the relevant energy levels in the structure and section $\mathrm{V}$ showing time resolved PL (TRPL) in order to characterize the decay constants of electrons in the device.

\section{Concept and Samples}

\subsection{Concept}

The key concept that we seek to exploit in the two structures to follow is that of energy selective tunneling from a narrow bandgap to a wider bandgap semiconductor. This mechanism, driven by a difference in electron temperature between the two regions, is the driving force for our cell. This method of realizing a hot carrier cell relies crucially on the transport of electrons from the absorber region to the collector region at a rate faster than they can thermalize with the phonon bath. This mechanism is shown schematically in figure 1 .

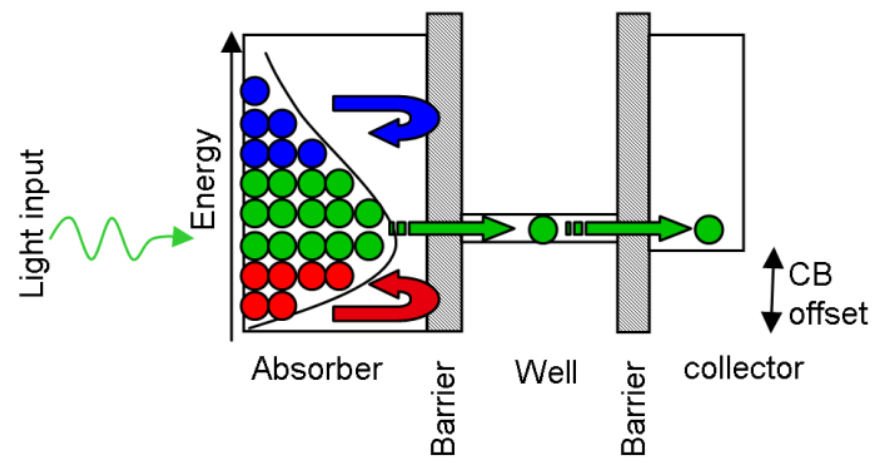

Figure. 1. The schematic conduction band structure showing energy selective tunneling from a narrow bandgap semiconductor (absorber) to a wider bandgap semiconductor (collector)

The rate of carrier transfer by tunneling through a double barrier quantum well region is strongly influenced by the barrier thickness, with thin barriers giving a wider transmission line width and consequently a faster speed of electron transfer [9]; therefore our structures have potential barriers of $2 \mathrm{~nm}$ thickness or less to achieve picosecond transfer rates. Previously we had theoretically justified our structural design and optoelectronically characterized the final device to show characteristics of hot carrier transfer [8]; in this paper we will further explore both fundamental and device properties, demonstrating improved IV characteristics and exploring the carrier transport and structural properties through optical measurements.

\subsection{Samples}

Samples to explore these concepts were grown by molecular beam epitaxy. Structure A is a p-i-n structure, used to assess IV characteristics and structure $B$ is an MQW structure used to investigate band structure and carrier transport properties through PL measurements.

\subsubsection{P-i-n structure}

The p-i-n structure was grown to study how an improved extraction for holes over the original n-i-n hot carrier structure [8] would increase the photocurrent and voltage of the cell.

The original structure was a n-i-n structure, this had the benefit of the sole driving force of the cell being the difference in the temperature and density of the electron populations of the absorber and collector, allowing us to unambiguously probe the hot carrier contribution to the device characteristics, but the drawback that this lowered efficiency. As parenthetically mentioned in the introduction a thermally driven current need not be the sole driving force for the cell, this current can supplement a field driven current, as is the aim with the p-i-n structure presented in this paper.

The $\mathrm{p}-\mathrm{i}-\mathrm{n}$ structure is structurally similar to our previously presented $n-i-n$ cell, with the difference that the top contacting layer is a $10 \mathrm{~nm} 5 \times 10^{17} \mathrm{~cm}^{-3} \mathrm{p}$-doped region of GaAs. The processing into mesas for final devices was also carried out as previously presented, with the exception that the metallization to the $\mathrm{p}-\mathrm{GaAs}$ region was $\mathrm{Au} / \mathrm{Zn} / \mathrm{Au}$. A schematic of the final processed p-i-n structure is shown in figure 2 .

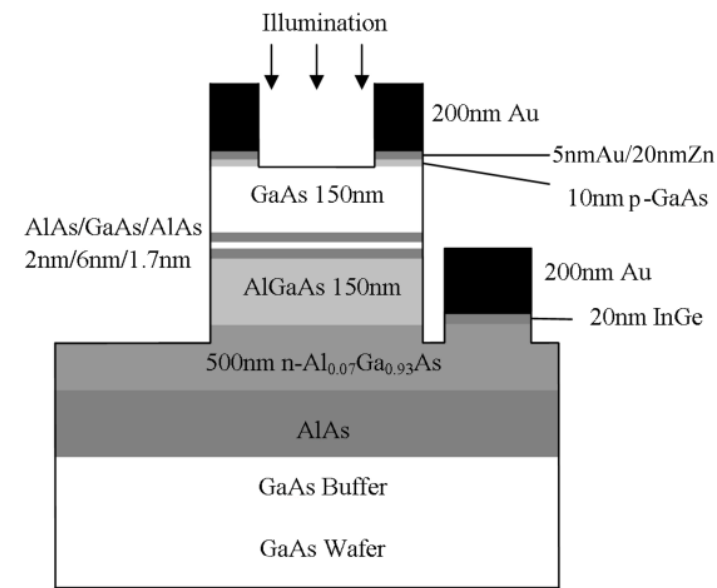

Figure. 2. A schematic illustration of the layer and processed mesa structure of the p-i-n device (structure A)

\subsubsection{MQW structure}

The MQW bandstructure is shown in figure 3 along with the wavefunctions for the three important states in this structure. The structure has a $10 \mathrm{~nm}$ p-doped GaAs cap and a $150 \mathrm{~nm}$ intrinsic GaAs absorber region, the last $5 \mathrm{~nm}$ of which is shown as the region between $155-160 \mathrm{~nm}$ in figure 3 . This region is followed by a $6.5 \mathrm{~nm}$ wide $\mathrm{GaAs} \mathrm{QW}$, confined by AlAs barriers. Up to this point structure A and structure B are identical; however, from this point while structure A has a bulk AlGaAs collector, structure $\mathrm{B}$ has a GaAs/AlGaAs MQW region followed by a bulk AlGaAs region. As illustrated in figure 3, our transfer matrix calculations predict that the $6.5 \mathrm{~nm}$ GaAs well gives rise to a transmissive (between absorber region and collector region) energy state which is $0.069 \mathrm{eV}$ above the GaAs conduction band minimum at the absorber/AlAs barrier (labelled $\Delta \mathrm{E}_{1}$ ); we will refer to this state and region as the "RTD" state and region, since it acts similarly to the transmissive tunneling state in a resonant tunneling diode. In addition to this state the MQW region gives two additional non-transmissive energy states, a higher energy state MQW+ and a lower energy state "MQW". These states are termed "non-transmissive", as they have a negligibly weak transmission with a very low tunneling probability between the MQW and RTD regions. Other states are also present, but only the states relevant to the following analysis are presented in figure 3 for clarity.

The aim of this structure is to provide signature PL emission from four regions of the device (GaAs absorber, 
RTD state, MQW state, and AlGaAs collector and barrier regions). The MQW+ state is important for the scattering of electrons from the RTD state but is not observed in PL, presumably due to rapid decay of carriers from MQW+ to the MQW state, so we only refer to PL from the MQW state. In addition to this signature region for PL examination, sample B was prepared by mounting on a glass cover-slip and removing the GaAs substrate up to an AlAs etch stop directly beneath the AlGaAs region; this allowed us to focus on the PL from the four regions previously mentioned and remove any impact from the substrate. The emission from these regions will be explored both in its temporal evolution and temperature dependence in order to reveal the structure of the device and show the nature of electron transport through it.

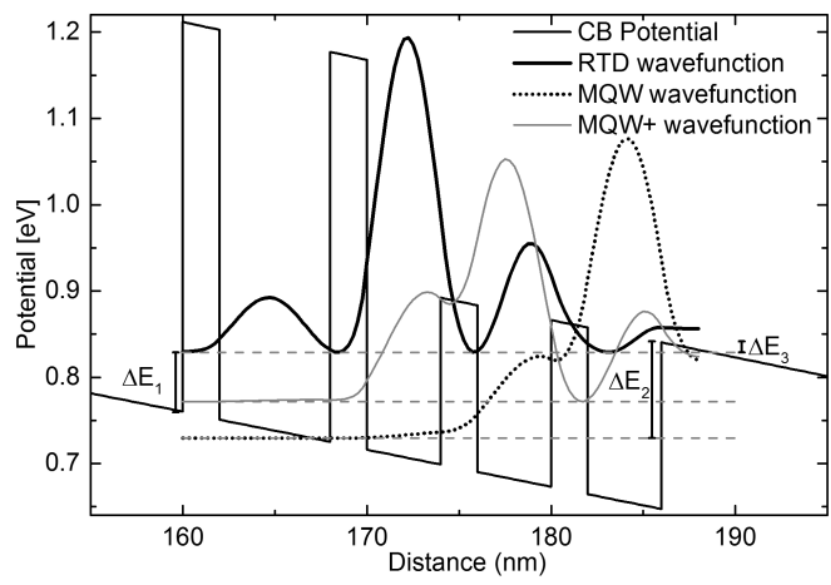

Figure. 3. The schematic band structure of the MQW sample with confined state wavefunctions overlaid as calculated by transfer matrix calculations. Activation energies for PL analysis are labelled as $\Delta \mathrm{E}_{1}=69 \mathrm{meV}$, $\Delta \mathrm{E}_{2}=111 \mathrm{meV}, \Delta \mathrm{E}_{3}=12 \mathrm{meV}$

\section{IV results}

Structure A was illuminated with monochromatic light at wavelengths from $805-815 \mathrm{~nm}$ and at temperatures from $88 \mathrm{~K}$ $293 \mathrm{~K}$. The illumination wavelengths and temperatures were chosen so as to excite carriers in the absorber region but not in the collector region or the confined regions.

Similarly to the previously presented $n-i-n$ device, structure A shows an IV curve characteristic of resonant tunneling, showing a pronounced peak and valley characteristic, figure 4 shows the IV characteristic of structure A under $805 \mathrm{~nm}$ illumination at a lattice temperature of $115 \mathrm{~K}$. For these experiments the illumination source was a wavelength tuneable Ti-sapphire laser (Millennia pumped Tsunami made by Spectra-Physics, Mountain View, CA, USA), focused to a spot size of $50 \mu \mathrm{m}$ with an objective lens. The laser wavelengths were measured with a calibrated Anritsu (Anritsu EMEA Ltd, Kanagawa 43-8555, Japan) MS9710C spectrum analyser. The structure was cooled on a Linkam (Linkam Scientific Instruments, Surrey, UK) THMSE600 cryogenic microscope stage attached to an LNP93 liquid nitrogen pump.

The main graph of figure 4 shows the dependence of the IV of structure A on the laser power (measured at source) normalized such that the current peak for each illumination power is equal to 1 to allow comparison between different powers of illumination.

The right hand axis of figure. 4 demonstrates the success of the p-i-n contacting of our device, showing the raw current density for $15 \mathrm{~mW}$ device illumation, showing that it is capable of sustaining the high current densities (over $3 \mathrm{~A} / \mathrm{cm}^{2}$ ) that a photovoltaic cell requires under concentrated illumination [10]. In addition to this high current density, the device also has a high $\mathrm{V}_{\mathrm{oc}}$, in excess of $1 \mathrm{~V}$ in comparison to the previously presented $n-i-n$ cell which had a $\mathrm{V}_{\mathrm{oc}}$ of $0.5 \mathrm{~V}$. The significantly higher $\mathrm{V}_{\mathrm{oc}}$ for the $\mathrm{p}-\mathrm{i}-\mathrm{n}$ structure is a result of the greater quasi-Fermi level separation between the absorber and collector, owing to the p-doping. In the $n-i-n$ structure the only driving force was the photogenerated electron temperature and density difference between the absorber and the collector, whereas in the p-i-n structure there is also a field driving electrons into the collector. In this way the $n-i-n$ structure is a clearer demonstration of a thermally driven current (since there is no other driving force present) while the $\mathrm{p}-\mathrm{i}-\mathrm{n}$ structure shows that this thermally driven current can supplement a field driven current and give rise to higher device performance. Given the mesa diameter of $15 \mathrm{um}$ we can use this JV to determine an approximate monochromatic efficiency at this wavelength and temperature of $1.6 \%$ for this device.

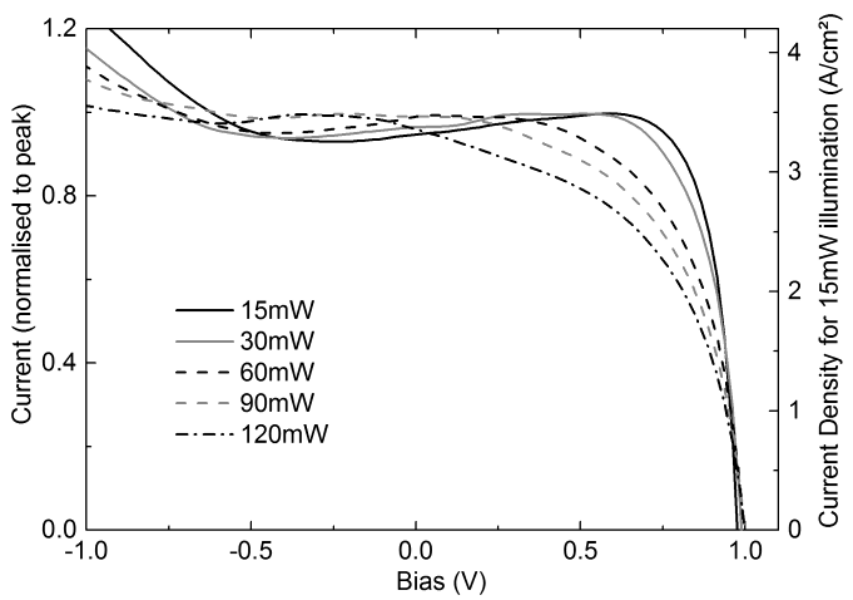

Figure. 4. Experimental IV characteristic of the p-i-n structure held at a temperature of $115 \mathrm{~K}$ and illuminated with $805 \mathrm{~nm}$ laser light of power between $15-120 \mathrm{~mW}$ focused on a 50um spot size. Left axis shows current of structure A normalized at 1 for current peak at all intensities, right axis shows absolute current density of structure A illuminated with $15 \mathrm{~mW}$ laser output power.

There is, however, a drawback with the p-i-n device in the dependence of the device characteristics on changes in light intensity that was not present in the $n-i-n$ device. The dependence on light intensity was explored as this is a key factor to control for when determining the hot carrier IV response of a cell to different wavelengths of light. Previously other authors [11]-[14] have shown strong hot carrier effects based on heating electron populations through increased light intensity, observing carrier temperature dependent features in the PL emission of their structures. However, this method of generating hot carriers (generating different temperatures with different illumination intensities) causes difficulty in analysis and interpretation when we examine the current from the structure rather than solely its optical emission. In particular, features that are caused by hot carrier effects can also be attributed to changes in electron density.

Previously we demonstrated the hot carrier characteristics of an n-i-n structure by showing a wavelength dependence of the current peak position of the IV curve [8], arguing that this showed hot carrier transport from the absorber to the collector and showed that this was not sensitive to changes in electron density. This was necessary to rule out fluctuations 
in photogenerated electron density contributing to any observed effect. The high sensitivity of the p-i-n sample to changes in light intensity, observed in figure 4 , means that analysing the wavelength dependence of the IV characteristic would be subject to much higher errors than the $n-i-n$ analysis.

The difference between the two devices' response to changes in light intensity arises from the change in position of the quasi-Fermi level for electrons under illumination. For the $n-i-n$ device absorption of light in the (n-doped) absorber region will not significantly alter the electron quasi-Fermi level, so the voltage response of the device is insensitive to changes in light intensity since the IV characteristic is determined by conduction band properties (i.e. tunneling from the electron population). By contrast, for the $\mathrm{p}-\mathrm{i}-\mathrm{n}$ device absorption of light in the (p-doped) absorber region will significantly alter the electron quasi Fermi-level. This change in the electron quasi-Fermi level as a function of illumination intensity gives rise to the peak shift that is observed in figure 4 , with higher intensities giving a current peak at more negative biases.

Because the light intensity has such a large effect on the IV properties, significantly larger than any hot carrier effects we might expect to observe through either intensity or wavelength variations, examining the IV characteristics is not helpful in discriminating any hot carrier effects for this structure; though they are likely to exist due to the structure's similarity to the n-i-n structure, for which such effects have been shown. As such, the investigation of $p-i-n$ hot carrier structures is continued in the following sections through purely optical means in order to explore the device structure and carrier transport.

\section{TDPL}

Structure B was designed to explore the carrier transport properties through the RTD state, which is a common feature across all our hot carrier photovoltaic structures, allowing for energy selective extraction from an absorber region to a collector region. In order to characterize the signature PL peaks of structure B we carried out TDPL on the sample, illuminating at $660 \mathrm{~nm}$ with a diode laser and varying the lattice temperature of the sample between $88-293 \mathrm{~K}$. The PL emission peaks were assigned by fitting their temperature dependent peak shift to the Varshni equation [15], with material dependent parameters obtained from the IOFFE

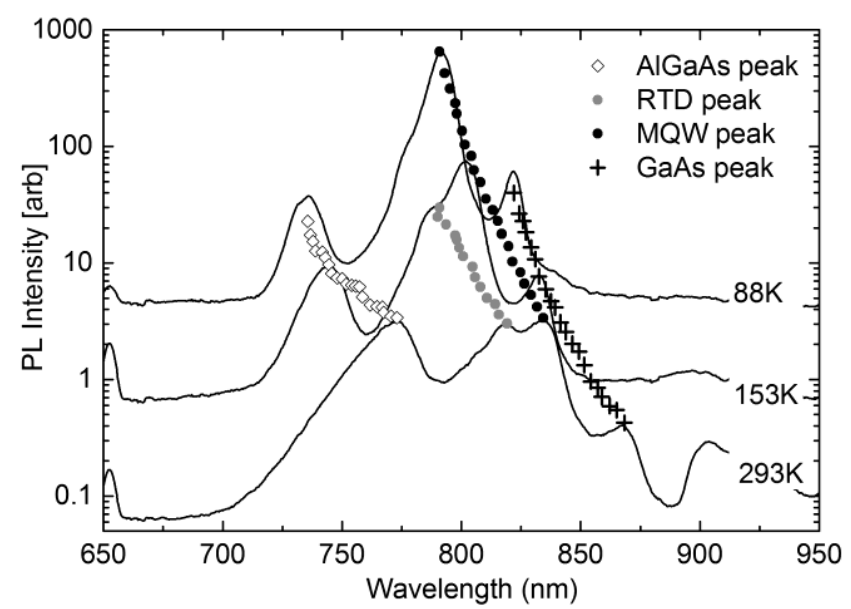

Figure. 5. PL spectra for temperatures from $88 \mathrm{~K} \rightarrow 293 \mathrm{~K}$, showing the low temperature quenching of the RTD peak relative to the MQW peak database [16] and previous characterisation. The rms errors in these fits were lower than $1 \%$.

Figure 5 shows three of the PL spectra obtained from structure B (at $88 \mathrm{~K}, 153 \mathrm{~K}$ and $293 \mathrm{~K}$ ) along with data points showing the assignment and location of the peaks at the other intermediate temperatures. An important feature of this set of spectra is the change in the relative intensity of the RTD and MQW peaks as temperature is decreased. At $293 \mathrm{~K}$ the MQW and RTD peaks are of approximately equal intensity, as expected from a transfer matrix analysis of the absorption through the structure. However, as the temperature is reduced the RTD peak is strongly quenched relative to the MQW peak, reducing to nearly an order of magnitude lower in intensity than the MQW peak at $88 \mathrm{~K}$. This feature can be explained by carriers scattering into the MQW state from the RTD state, this scattering will presumably occur via the MQW+ state since these states are each separated by a little over 1LO phonon in energy and have a strong wavefunction overlap. The temperature dependence of this quenching is important in the analysis of the TRPL data in section $\mathrm{V}$ and will be investigated further by plotting the integrated PL of each peak in Figure 5 as a function of temperature in an Arrhenius plot.

Figure 6 is an Arrhenius plot of the integrated PL of the GaAs, MQW and RTD regions, calculated from Gaussian fits to the peaks in the spectra shown in figure 5. An Arrhenius equation representing the temperature dependence of the luminescence is then fitted to each plot with a least squares fit, revealing the activation energy required to quench the PL for each region [17]. The most important feature of figure 6 is the activation energy for the GaAs peak, which is experimentally observed to be $74 \mathrm{meV}$, within $5 \mathrm{meV}$ of the calculated value for the RTD transmissive state relative to the GaAs $\mathrm{CB}$ minimum ( $\Delta \mathrm{E}_{1}$ in figure 3$)$. This is a strong indication that the quenching of the PL from the GaAs region is occurring through carriers transferring from the GaAs absorber through the RTD state, this will be further elaborated upon in section 5 , in which the rate of the PL decay in this region is examined and it is determined whether this rate is compatible with tunneling transfer and therefore hot carrier extraction as previously proposed.

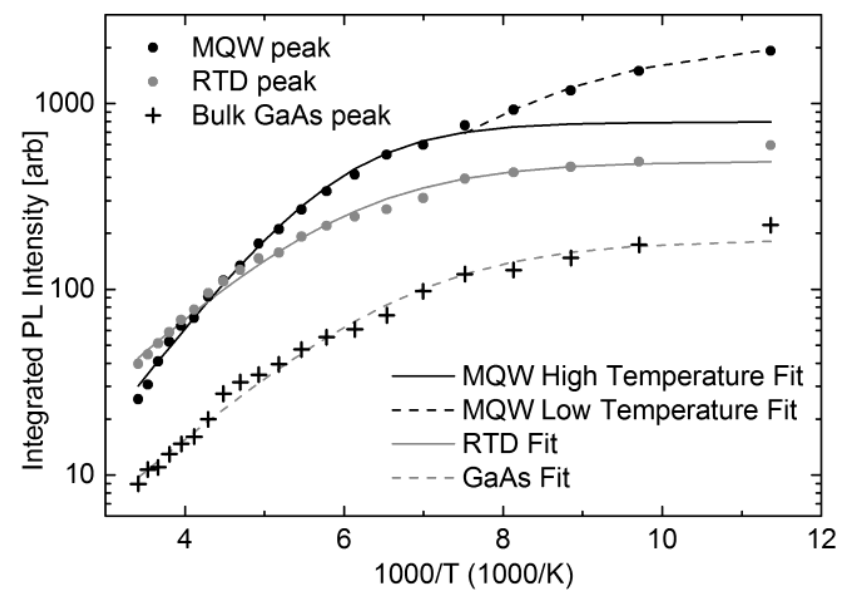

Figure. 6. Arrhenius plot of integrated PL vs temperature for the key peaks

In addition to this important feature, a second feature of Figure 6 reveals further information suggesting the reason behind the temperature dependence of the quenching of the RTD peak relative to the MQW peak. The integrated PL of the MQW peak shows different behaviour either side of a critical temperature of approximately $140 \mathrm{~K}$, showing a 
transition to a higher activation energy required to quench the PL at higher temperatures (solid black line) than lower temperatures (dashed black line). This behaviour can be explained, with reference to figure 3 , as resulting from electrons in the RTD state in the MQW region (between 180$186 \mathrm{~nm}$ in figure 3 ) being able to be thermally excited out of the RTD state and into the AlGaAs continuum at higher temperatures. Our bandstructure calculations presented in figure 3 show that the RTD state is $12 \mathrm{meV}$ below the AlGaAs continuum ( $\Delta \mathrm{E}_{3}$ in figure 3$)$, meaning that for temperatures in excess of $140 \mathrm{~K}$ carriers are able to be thermally excited from this region rather than decaying into the bound MQW state. This mechanism results in the behaviour observed in figure 5, whereby at low temperatures carriers scatter from the RTD state into the MQW state quenching the RTD PL relative to the MQW PL, whereas at high temperatures this does not occur. It also means that carriers cannot escape from the MQW state via the RTD state at higher temperatures, but must go directly to the continuum, resulting in a higher activation energy $\left(\Delta \mathrm{E}_{2}=110 \mathrm{meV}\right.$ in figure 3 , agreeing exactly with the experimentally determined activation energy) for PL quenching at high temperatures for the MQW PL.

The two features revealed through the TDPL analysis, GaAs PL quenching from carrier escape through the RTD state and the temperature dependence of carrier transfer from the RTD to the MQW state, are important for the device operation of the hot carrier photovoltaic cell and are built upon further in section $\mathrm{V}$ to reveal their time dependence. In particular, due to the thermal escape from the RTD state to the continuum, this device only operates as designed below $140 \mathrm{~K}$.

\section{Carrier dynamics of structure $B$ explored with TRPL}

In order to further explore the carrier transport properties in structure B TRPL was carried out at temperatures from $10 \mathrm{~K}$ $270 \mathrm{~K}$ using a Ti-sapphire laser at $720 \mathrm{~nm}$ and with a pulse width of $30 \mathrm{ps}$ to excite the sample. The sample was cooled in a closed cycle helium cryostat and the time resolved PL spectra were recorded on a Hamamatsu streak camera.

Figure. 7 shows the normalized peak intensities for the GaAs, RTD and MQW PL emission as a function of time at a lattice temperature of $10 \mathrm{~K}$. The time dependence of these peaks follows our expectations from section IV, with a very fast

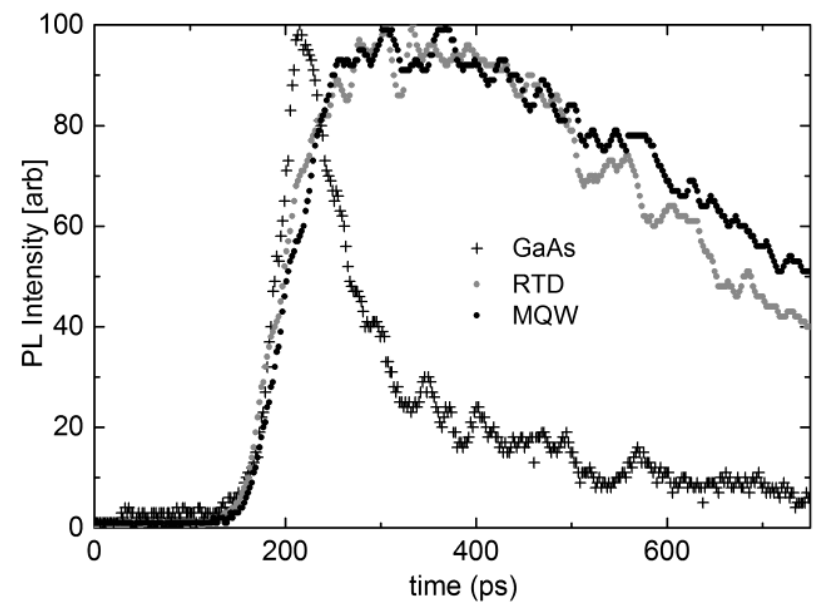

Figure. 7. Time resolved photoluminescence data at $10 \mathrm{~K}$ for the peak intensity as a function of time for the GaAs, RTD and MQW peaks decay for the GaAs PL giving further evidence to the proposed quenching mechanism of tunneling through the RTD state. In comparison with the GaAs PL decay, the RTD and MQW states decay significantly more slowly as one would expect for radiative recombination in these regions. De-convoluting the laser signal from the GaAs signal reveals that the characteristic decay time for PL from this region is 10ps; combined with the evidence presented in section 4 this indicates carrier escape from the GaAs region through the RTD state via tunneling. This is the designed mode of operation for our hot carrier cells, required for energy selective extraction, and it is on a timescale that is competitive with hot carrier decay in the GaAs region [18].

Figure 7 also reveals a small time difference between the peak PL observed in the GaAs region and the peak PL observed in the MQW and RTD regions. It also shows periodic oscillations in the PL decay from these regions with a period of $30-40 \mathrm{ps}$, which vanish at temperatures above $70 \mathrm{~K}$, suggestive of some oscillatory charge transfer [19]; though this is unlikely to be coherent due to the long timescales. In order to investigate this further we fitted a bigaussian equation to the TRPL data (to smooth over the periodic oscillations) and extracted the time difference (dt) between the GaAs PL peak intensity and the peak intensity for the MQW and RTD peaks over all the temperatures studied. This data is shown in figure 8 , revealing a constant temporal offset between the RTD and MQW PL peak intensity at low temperature, and between both these peaks and the GaAs peak intensity. This is further support for the suggested mode of operation of the cell, which is transfer of carriers from the GaAs via the RTD state followed by scattering from the RTD state into the MQW state.

Figure 8 shows that this scattering occurs on a timescale of $30-40$ ps, agreeing with the TRPL decay oscillation period, which would result in the quenching of the RTD PL already observed in figure 5. This total scattering time from RTD state to MQW state is long relative to the expected direct scattering time (approximately the LO phonon emission time). This is probably attributable to scattering occurring via the MQW+ state, since the overlap integral of the MQW+ and RTD wavefunctions is over four times larger than the overlap integral of the MQW and RTD wavefunctions. Further evidence for this carrier transfer from RTD to MQW can be seen in figure 7 in the faster decay of the PL from the RTD state than the MQW state. This difference in PL decay also shows a similar dependence on temperature to that

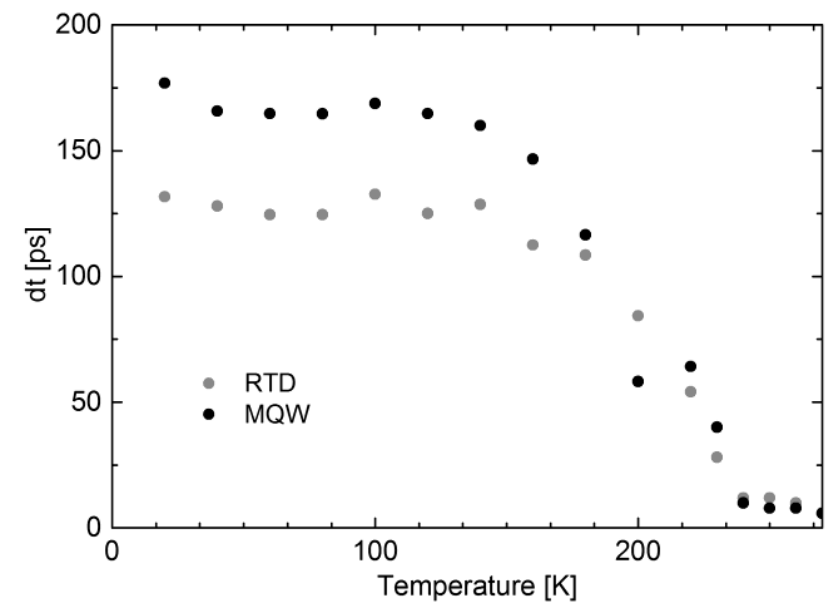

Figure. 8. Time difference between peak PL intensity of GaAs peak and RTD peak (grey) and MQW peak (black) 
observed in the peak PL intensity shown in figure 8 .

Importantly the dt between the GaAs and RTD and MQW states reduces once the temperature exceeds $140 \mathrm{~K}$, tending towards zero; again supporting the interpretation that above $140 \mathrm{~K}$ carriers are no longer confined in the RTD state in the MQW region and thus the majority of PL from the MQW and RTD regions at higher temperatures is attributable to direct photoexcitation in these regions.

\section{Conclusions}

The TDPL and TRPL data presented in sections IV and V has demonstrated that carriers are photogenerated in the GaAs absorber region of these structures and are extracted from this region via resonant tunneling on a timescale of $10 \mathrm{ps}$, confirming the hot carrier photovoltaic device operation presented previously [8]. Both TDPL and TRPL data support the mode of operation such that at lattice temperatures below $140 \mathrm{~K}$ carriers are extracted from the absorber region via a transmissive state and are scattered into a non-transmissive state. This temperature could be increased by further optimization of barrier heights. The fast extraction via tunneling is a requisite for hot carrier photovoltaic operation, allowing extraction of carriers before they thermalize. The transmission via a transmissive state followed by scattering into a non-transmissive state was used primarily as a signature to investigate the carrier dynamics in our cell, but could also be an important feature of an improved hot carrier cell, providing some directionality to the device, akin to a ratchet [20].

In addition to these optical assessments we have shown a low temperature IV characteristic of an improved prototype structure, showing that such a cell supports a photocurrent density greater than $3 \mathrm{~A} / \mathrm{cm}^{2}$ and has a $\mathrm{V}_{\mathrm{oc}}$ in excess of $1 \mathrm{~V}$.

\section{Acknowledgments}

J. Dimmock gratefully acknowledges a Royal Commission for the Exhibition of 1851 Industrial Fellowship.

\section{References}

[1] S. Kettemann and J.-F. Guillemoles, "Thermoelectric field effects in low-dimensional structure solar cells," Phys. E Low-Dimens. Syst. Nanostructures, vol. 14, no. 1-2, pp. 101-106, Apr. 2002.

[2] P. Würfel, "Solar energy conversion with hot electrons from impact ionisation," Sol. Energy Mater. Sol. Cells, vol. 46, no. 1, pp. 43-52, 1997.

[3] N.-P. Harder and P. Würfel, "Theoretical limits of thermophotovoltaic solar energy conversion," Semicond. Sci. Technol., vol. 18, no. 5, p. S151, 2003.

[4] A. Le Bris and J.-F. Guillemoles, "Hot carrier solar cells: Achievable efficiency accounting for heat losses in the absorber and through contacts," Appl. Phys. Lett., vol. 97, no. 11, p. 113506, 2010.

[5] L. C. Hirst, H. Fujii, Y. Wang, M. Sugiyama, and N. J. Ekins-Daukes, "Hot Carriers in Quantum Wells for Photovoltaic Efficiency Enhancement," IEEE J. Photovolt., vol. 4, no. 1, pp. 244-252, Jan. 2014.

[6] S. K. Shrestha, S. Chung, N. Gupta, Y. Feng, X. Wen,
H. Xia, and G. J. Conibeer, "Evaluation of Hafnium Nitride and Zirconium Nitride as Hot Carrier Absorber," in Proceedings of the 40th IEEE Photovoltaic Specialists Conference, Denver, CO, 2014.

[7] Y. Yao and D. König, "Comparison of bulk material candidates for hot carrier absorber," Sol. Energy Mater. Sol. Cells, vol. 140, pp. 422-427, Sep. 2015.

[8] J. A. R. Dimmock, S. Day, M. Kauer, K. Smith, and J. Heffernan, "Demonstration of a hot-carrier photovoltaic cell," Prog. Photovolt. Res. Appl., vol. 22, no. 2, pp. 151-160, Feb. 2014.

[9] P. J. Price, "Theory of resonant tunneling in heterostructures," Phys. Rev. B, vol. 38, no. 3, pp. 1994-1998, Jul. 1988.

[10] D. Masson, F. Proulx, and S. Fafard, "Pushing the limits of concentrated photovoltaic solar cell tunnel junctions in novel high-efficiency GaAs phototransducers based on a vertical epitaxial heterostructure architecture," Prog. Photovolt. Res. Appl., vol. 23, no. 12, pp. 1687-1696, Dec. 2015.

[11] A. Le Bris, L. Lombez, S. Laribi, G. Boissier, P. Christol, and J.-F. Guillemoles, "Thermalisation rate study of GaSb-based heterostructures by continuous wave photoluminescence and their potential as hot carrier solar cell absorbers," Energy Environ. Sci., vol. 5, no. 3, p. 6225, 2012.

[12] L. C. Hirst, R. J. Walters, M. F. Führer, and N. J. Ekins-Daukes, "Experimental demonstration of hotcarrier photo-current in an InGaAs quantum well solar cell," Appl. Phys. Lett., vol. 104, no. 23, p. 231115 , Jun. 2014.

[13] J. Tang, V. R. Whiteside, H. Esmaielpour, S. Vijeyaragunathan, T. D. Mishima, M. B. Santos, and I. R. Sellers, "Effects of localization on hot carriers in InAs/AlAsxSb1-x quantum wells," Appl. Phys. Lett., vol. 106, no. 6, p. 061902, Feb. 2015.

[14] J. Rodière, L. Lombez, A. L. Corre, O. Durand, and J.F. Guillemoles, "Experimental evidence of hot carriers solar cell operation in multi-quantum wells heterostructures," Appl. Phys. Lett., vol. 106, no. 18, p. 183901, May 2015.

[15] Y. P. Varshni, "Temperature dependence of the energy gap in semiconductors," Physica, vol. 34, no. 1, pp. 149-154, 1967.

[16] "IOFFE Semiconductor Database," http://www.ioffe.ru/SVA/NSM/Semicond/index.html. .

[17] E. C. Le Ru, J. Fack, and R. Murray, "Temperature and excitation density dependence of the photoluminescence from annealed InAs/GaAs quantum dots," Phys. Rev. B, vol. 67, no. 24, p. 245318, Jun. 2003.

[18] Y. Rosenwaks, M. C. Hanna, D. H. Levi, D. M. Szmyd, R. K. Ahrenkiel, and A. J. Nozik, "Hot-carrier cooling in GaAs: Quantum wells versus bulk," Phys. Rev. B, vol. 48, no. 19, pp. 14675-14678, Nov. 1993.

[19] J. Shah, Ultrafast Spectroscopy of Semiconductors and Semiconductor Nanostructures. Springer Science \& Business Media, 2013.

[20] M. Yoshida, N. J. Ekins-Daukes, D. J. Farrell, and C. C. Phillips, "Photon ratchet intermediate band solar cells," Appl. Phys. Lett., vol. 100, no. 26, p. 263902 , Jun. 2012. 